\title{
Dynamics of a Delayed Solow Model with Effective Labor Demand
}

\author{
Sanaa ElFadily ${ }^{*}$, Abdelilah Kaddar ${ }^{2}$ and Khalid Najib ${ }^{3}$ \\ ${ }^{1}$ Mohammed V University in Rabat, Mohammadia School of engineering, Rabat, Morocco \\ ${ }^{2}$ Faculty of Juridical, Economic and Social Sciences of Salé, Mohammed V University in Rabat \\ ${ }^{3}$ Ecole Nationale Supérieure des Mines de Rabat \\ Email: a.kaddar@yahoo.fr
}

\begin{abstract}
In this paper, we propose a model of mutual interactions between the economically active population and the economic growth. Our principal goal is to introduce a delayed equation of the active population evolution. The time delay, resulting from the recruitment processes, is incorporated in the effective labor demand. The dynamics are studied in terms of local stability and of the description of the Hopf bifurcation, is proven to exist as the delay (taken as a parameter of bifurcation) cross some critical value. Some numerical simulations are given to illustrate our theoretical results. Additionally we conclude with some remarks.
\end{abstract}

Keywords: Solow model, economic growth, effective labor demand, delayed differential equations, stability, Hopf bifurcation.

\section{Introduction}

Economic growth corresponds to positive change in gross domestic product (GDP), over a period of one year. This change depends on the use of production factors: capital, labor and total factor productivity. The capital refers to resources used to create goods or services that are not themselves significantly consumed in the production process. The labor is related to the workforce. It increases levels of economic growth by increasing the number of hours work and by improving the quality of this work. Finally, the total factor productivity refers to anything that is not explained by the above two factors, we cite for example: the organizational innovation and technological innovation.

Theoretically, the increase of capital stock, the workforce and the qualitative improvement of these factors are essential components and engines of economic growth. Economists have long examined the effect of the change in these factors on the economic growth. For example, the effect of variation of active population on the economic growth. These studies have led to three conclusions: The first conclusion states that population growth stimulates economic growth. The second view indicates that population growth adversely affects economic growth and the third point of view asserts that population growth is a neutral factor in economic growth.

In this work, we will try to model the phenomenon of mutual interactions between economically active population and economic growth. Our starting point is based on three models: the classical Solow model of economic growth (1956, [1]), the active population growth model (logistic model) introduced by Hallegatte et al. (2008, [2]) and the economic growth model with a carrying capacity proposed by Cai (2012, [3]). The resulting model is the following delay differential system:

$$
\left\{\begin{array}{l}
\frac{d K}{d t}=f(K, L)-\delta K \\
\frac{d L}{d t}=\gamma L\left[1-\frac{L_{\tau}}{g\left(K_{\tau}\right)}\right]
\end{array}\right.
$$

where $K$ is the capital stock, $L$ is the number of employed workers, $\delta$ is the depreciation rate of capital stock, $f$ is the production function, $g$ is the effective labor demand and $\tau$ is the time needed to assess needs for labor force and the time taken for the recruitment of this labor force.

We assume that the function $g$ is continuously differentiable, satisfying the following hypotheses:

$\left(H_{1}\right): g(0)>0$ 
$\left(H_{2}\right): g$ is a strictly monotone increasing and concave function;

$\left(H_{3}\right): \lim _{K \rightarrow+\infty} g(K)=L_{e}$;

where $L_{e}$ is the maximal effective labor demand [2]. Moreover, as in the Solow model [1], we consider a Cobb-Douglas function [4]:

$$
f(K, L)=s A K^{\alpha} L^{1-\alpha},
$$

where $s$ denotes the constant saving rate, $A$ is a positive constant that reflects the level of the technology and $\alpha \in(0,1)$ is a constant.

The first model in this is the Harrod-Domar model, presented by Harrod (1939, [5]) and completed by Domar $(1947,[6])$. This keynesian model stresses the importance of savings and investments as key determinants of growth.

Based on the Harrod-Domar model, Solow presented the following new-classical growth model (1956, [1]):

$$
\frac{d K}{d t}=s A K^{\alpha} L^{1-\alpha}-\delta K
$$

This model attempts to explain long-run economic growth by considering a capital accumulation, labor growth, and technological progress.

In (2010, [7]), Cai integrated the logistically variable population into the Solow model and he proved the existence of the saddle-node bifurcations.

In $(2013,[8])$, Guerrini and Sodini assumed that population of workers evolves according to the delayed logistic equation with constant carrying capacity as follows:

$$
\left\{\begin{array}{c}
\frac{d K}{d t}=s A K\left(t-\sigma_{1}\right)^{\alpha} L(t)^{1-\alpha}-\delta K(t), \\
\frac{d L}{d t}=\gamma L(t)\left[a-b L\left(t-\sigma_{2}\right)\right],
\end{array}\right.
$$

where $K$ and $L$ are capital and labor force, respectively, $\frac{a}{b}$ is the constant carrying capacity, $\sigma_{1}$ is the time delay between new investment and production (Kalecki's time delay [9]) and $\sigma_{2}$ is the time delay between the birth date and the recruitment in the labor force.

For $\sigma_{1}=\sigma_{2}$, the authors showed that the coexistence of delays is a source of cyclical behavior in capital accumulation.

In $(2013,[10])$, Guerrini proposed the following generalization of the model (2):

$$
\left\{\begin{array}{c}
\frac{d K}{d t}=s A K(t-\sigma)^{\alpha} N(t)^{1-\alpha}-\delta K(t-\sigma) \\
\frac{d N}{d t}=\gamma N(t)\left[1-\frac{N(t)}{g(K(t))}\right]
\end{array}\right.
$$

where $N$ is the population size, $\gamma$ is a reproduction rate, $\sigma$ is the Kalecki's time delay and $g$ is the carrying capacity.

In this model, Guerrini assumed that there is full employment in the economy, so that employment and labor supply coincide, i.e. $L=N$. He proved that the system (3) loses stability and a Hopf bifurcation occurs when the time delay passes through critical values.

In this work, we assume that economy is not at full employment $(L<N)$ and we show that a time delay in recruitment process can destabilize the system giving birth to economic fluctuations. The dynamics of the system (1) are studied in terms of local stability and of the description of the Hopf bifurcation, is proven to exist as the delay (taken as a parameter of bifurcation) cross some critical value. In the end some numerical simulations are given to illustrate our theoretical results.

\section{$2 \quad$ Stability Analysis and Hopf Bifurcation Occurrence}

In this section, we discuss the local asymptotic stability of the positive equilibrium of the system (1). In order to do this, we give a sufficient conditions for the existence and uniqueness of a positive equilibrium $\left(K_{*}, L_{*}\right)$. 


\subsection{Steady State}

Proposition 1 System (1) always has two equilibria $P_{0}=(0,0)$ and $P_{1}=(0, g(0))$ which exist for all parameter values. On the other hand, if hypotheses $\left(H_{1}\right),\left(H_{2}\right)$ and $\left(H_{3}\right)$ hold, then system (1) also admits a unique positive equilibrium $\left(K_{*}, L_{*}\right)$, where $K_{*}$ is the unique positive solution of

$$
g(K)=\left(\frac{\delta}{s A}\right)^{\frac{1}{1-\alpha}} K
$$

and $L_{*}$ is determined by

$$
L_{*}=\left(\frac{\delta}{s A}\right)^{\frac{1}{1-\alpha}} K_{*}
$$

Proof. $(K, L)$ is an equilibrium of (1) if

that is

$$
\frac{d K}{d t}=\frac{d L}{d t}=0
$$

$$
\left\{\begin{array}{c}
s A K^{\alpha} L^{1-\alpha}-\delta K=0 \\
\gamma L\left[1-\frac{L_{\tau}}{g\left(K_{\tau}\right)}\right]=0
\end{array}\right.
$$

It's easy to see that equation (6) has two trivial solutions: $(0,0)$ and $(0, g(0))$.

Now, let us assume that $K>0$ and $L>0$ satisfy (6). Then

$$
L=\left(\frac{\delta}{s A}\right)^{\frac{1}{1-\alpha}} K
$$

and

$$
g(K)=\left(\frac{\delta}{s A}\right)^{\frac{1}{1-\alpha}} K .
$$

In view of hypotheses $\left(H_{1}\right),\left(H_{2}\right)$ and $\left(H_{3}\right)$, it's clear that equation (8) has a unique solution $K_{*}>0$. This concludes the proof.

\subsection{Local Stability Analysis}

Let $x=K-K_{*}$ and $y=L-L_{*}$. Then by linearizing system $(1)$ around $\left(K^{*}, L^{*}\right)$ we have

$$
\left\{\begin{array}{c}
\frac{d K}{d t}=\left[\frac{\partial f}{\partial K}\left(K_{*}, L_{*}\right)-\delta\right]\left(K-K_{*}\right)+\frac{\partial f}{\partial L}\left(K_{*}, L_{*}\right)\left(L-L_{*}\right), \\
\frac{d L}{d t}=-\gamma L_{*}^{2}\left(\frac{-g^{\prime}\left(K_{*}\right)}{g^{2}\left(K_{*}\right)}\right)\left(K-K_{*}\right)+\gamma L_{*}^{2}\left(\frac{-1}{g\left(K_{*}\right)}\right)\left(L-L_{*}\right),
\end{array}\right.
$$

where $f(K, L)=s A K^{\alpha} L^{1-\alpha}$.

According to (4) and (5), we get

$$
\left\{\begin{array}{c}
\frac{d x}{d t}=(\alpha-1) \delta x+(1-\alpha) \delta K_{*} L_{*}^{-1} y \\
\frac{d y}{d t}=\gamma g^{\prime}\left(K_{*}\right) x_{\tau}-\gamma y
\end{array}\right.
$$

The characteristic equation associated to system (10) is

$$
\lambda^{2}+a \lambda+b \lambda \exp (-\lambda \tau)+c+d \exp (-\lambda \tau)=0,
$$

where

$$
\begin{gathered}
a=\delta(1-\alpha), \\
b=\gamma, \\
c=0,
\end{gathered}
$$

and

$$
d=\gamma \delta(1-\alpha)(1-M)
$$

where $M=K_{*} L_{*}^{-1} g^{\prime}\left(K_{*}\right)$. 
The local stability of the steady state $\left(K_{*}, L_{*}\right)$ is a result of the localization of the roots of the characteristic equation (11). In order to investigate the local stability of the steady state, we begin by considering the case without delay $\tau=0$. In this case the characteristic equation (11) reads as

$$
\lambda^{2}+(\gamma+\delta(1-\alpha)) \lambda+\gamma \delta(1-\alpha)(1-M)=0
$$

hence, according to the Hurwitz criterion, we have the following lemma.

Lemma 2 For $\tau=0$, the equilibrium $\left(K_{*}, L_{*}\right)$ is locally asymptotically stable if and only if $M<1$.

We now return to the study of equation (11) with $\tau>0$.

Theorem 3 If $M<1$, then there exists $\tau_{0}>0$ such that,

(i) for $\tau \in\left[0, \tau_{0}\right)$ the steady state $\left(K_{*}, L_{*}\right)$ is locally asymptotically stable;

(ii) for $\tau>\tau_{0},\left(K_{*}, L_{*}\right)$ is unstable;

(iii) for $\tau=\tau_{0}$, equation (11) has a pair of purely imaginary roots $\pm i \omega_{0}$;

with

$$
\omega_{0}^{2}=\frac{1}{2}\left\{\left(\gamma^{2}-((1-\alpha) \delta)^{2}\right)+\left[\left(\gamma^{2}-((1-\alpha) \delta)^{2}\right)^{2}-4 \delta^{2} \gamma^{2}(1-\alpha)^{2}(1-M)^{2}\right]^{\frac{1}{2}}\right\},
$$

and

$$
\tau_{0}=\frac{1}{\omega_{0}} \arccos \frac{\omega_{0}^{2} \delta(\alpha-1) M}{\gamma\left(\omega_{0}^{2}+(\delta(1-\alpha)(1-M))^{2}\right.} .
$$

Proof. From the hypothesis $M<1$, the characteristic equation (11) has negative real parts for $\tau=0$ (see lemma 2.1). By Rouche's theorem ([11], p.248), it follows that if instability occurs for a particular value of the delay $\tau$, a characteristic root of (11) must intersect the imaginary axis. Suppose that (11) has a purely imaginary root $i \omega$, with $\omega>0$. Then, by separating real and imaginary parts in (11), we have

$$
\left\{\begin{array}{c}
-\omega^{2}+\delta \gamma(1-\alpha)(1-M) \cos (\omega \tau)+\omega \gamma \sin (\omega \tau)=0 \\
(1-\alpha) \delta \omega+\omega \gamma \cos (\omega \tau)-\delta \gamma(1-\alpha)(1-M) \sin (\omega \tau)=0
\end{array}\right.
$$

Hence,

$$
\omega^{4}+\left((1-\alpha)^{2} \delta^{2}-\gamma^{2}\right) \omega^{2}+\delta \gamma(1-\alpha)(1-M)=0
$$

It's roots are

$$
\omega_{ \pm}^{2}=\frac{1}{2}\left\{\gamma^{2}-\left((1-\alpha)^{2} \delta\right)^{2}+\left[\left(\gamma^{2}-(1-\alpha) \delta^{2}\right)^{2}-4 \delta^{2} \gamma^{2}(1-\alpha)^{2}(1-M)^{2}\right]^{1 / 2}\right\} .
$$

Clearly, the hypothesis $M<1$ implies that $\omega_{0}=\omega_{+}$makes sense.

From equations (13), we obtain the following set of values of $\tau$ for which there are imaginary roots:

$$
\tau_{n, 1}=\frac{\theta_{1}}{\omega_{0}}+\frac{2 n \pi}{\omega_{0}},
$$

where $0 \leq \theta_{1}<2 \pi$, and

$$
\cos \theta_{1}=\frac{\omega_{0}^{2} \delta(\alpha-1) M}{\gamma^{2} \omega_{0}^{2}+(\delta(1-\alpha)(1-M))^{2}}
$$

where $n=0,1,2, \ldots$

We set

$$
\tau_{0}=\tau_{0,1} .
$$

Thus, we have: if $\tau \in\left[0, \tau_{0}\right)$, then $\left(K_{*}, L_{*}\right)$ is locally asymptotically stable. If $\tau>\tau_{0}$, then $\left(K_{*}, L_{*}\right)$ is unstable and if $\tau=\tau_{0}$, then equation (11) has a purely imaginary roots $\lambda_{0}= \pm i \omega_{0}$. 


\subsection{Hopf Bifurcation Occurrence}

According to the Hopf bifurcation theorem [12], we establish sufficient conditions for the local existence of periodic solutions.

Theorem 4 Under hypothesis $M<1$, a Hopf bifurcation of periodic solutions of system (1) occurs at $\left(K_{*}, L_{*}\right)$ when $\tau=\tau_{0}$.

Proof. For the proof of this theorem we apply the Hopf bifurcation theorem (see, for example [12]). From Theorem 2.2, the characteristic equation (11) has a pair of imaginary roots $\pm i \omega_{0}$ at $\tau=\tau_{0}$. In the first, lets show that $i \omega_{0}$ is simple: Consider the branch of characteristic roots $\lambda(\tau)=\nu(\tau)+i \omega(\tau)$, of equation (11) bifurcating from $i \omega_{0}$ at $\tau=\tau_{0}$. By derivation of (11) with respect to the delay $\tau$, we obtain

$$
\left\{2 \lambda+\delta(1-\alpha)[\gamma-\tau(\gamma \lambda+\delta \gamma(1-\alpha)(1-M))] e^{-\lambda \tau}\right\} \frac{d \lambda}{d \tau}=\lambda \gamma(\lambda+\delta(1-\alpha)(1-M)) e^{-\lambda \tau}
$$

If we suppose, by contradiction, that $i \omega_{0}$ is not simple, the right hand side of (16) gives

$$
\delta(1-\alpha)(1-M))+i \omega_{0}=0,
$$

and leads to a contradiction with the fact that $\omega_{0}>0$.

Lastly we need to verify the condition,

$$
\frac{d R e(\lambda)}{d \tau} \mid \tau_{0} \neq 0
$$

From (16), we have

$$
\left(\frac{d \lambda}{d \tau}\right)^{-1}=\frac{(2 \lambda+\delta(1-\alpha)) \exp (\lambda \tau)+\gamma}{\gamma \lambda(\lambda+\delta(1-\alpha)(1-M))}-\frac{\tau}{\lambda} .
$$

From (11), we have

$$
\exp (\lambda \tau)=-\frac{\gamma(\lambda+\delta(1-\alpha)(1-M))}{\lambda^{2} \delta(1-\alpha) \lambda}
$$

As,

$$
\operatorname{Sign}\left\{\frac{d R e(\lambda)}{d \tau} \mid \tau_{0}\right\}=\operatorname{Sign}\left\{\operatorname{Re}\left(\frac{d \lambda}{d \tau}\right)^{-1} \mid \tau_{0}\right\}
$$

Then

Thus, we obtain

$$
\operatorname{Sign} \frac{d \operatorname{Re}(\lambda)}{d \tau} \mid \tau_{0}=\operatorname{Sign}\left\{\operatorname{Re} \frac{-2(\lambda+(1-\alpha) \delta)}{(1-\alpha) \delta+\lambda) \lambda^{2}}+\operatorname{Re} \frac{1}{\lambda(\lambda+\delta(1-\alpha))(1-M)}\right\}
$$

$$
\begin{aligned}
\operatorname{Sign}\left\{\frac{d R e(\lambda)}{d \tau} \mid \tau_{0}\right\} & =\operatorname{Sign}\left\{\frac{2 \omega_{0}^{2}+(\delta(1-\alpha))^{2}}{\omega_{0}^{2}\left(\delta^{2}(1-\alpha)^{2}+\omega_{0}^{2}\right)}-\frac{1}{\gamma^{2}\left(\omega_{0}^{2}+\delta^{2}(1-\alpha)^{2}(1-M)^{2}\right)}\right\} \\
& =\operatorname{Sign}\left\{\left(\delta^{2}(1-\alpha)\right)^{2}-\gamma^{2}+2 \omega_{0}^{2}\right\}
\end{aligned}
$$

By inserting the expression for $\omega_{0}^{2}$, we obtain:

$$
\operatorname{Sign}\left\{\frac{d \operatorname{Re}(\lambda)}{d \tau} \mid \tau_{0}\right\}=\operatorname{Sign}\left\{\left(\gamma^{2}-\delta^{2}(1-\alpha)\right)^{2}+4 \gamma^{2} \delta^{2}(1-\alpha)^{2}(1-M)^{2}\right\}
$$

Hence

$$
\frac{d R e(\lambda)}{d \tau}\left(\tau_{0}\right)>0
$$

Proposition 5 if $M=1$ and $\delta(1-\alpha) \geq \gamma$, then the steady state $\left(K_{*}, L_{*}\right)$ is stable, but not asymptotically stable.

Proof. if $M=1$ and $\delta(1-\alpha) \geq \gamma$, all roots of characteristic equation (11) have non positive real parts, this implies that $\left(K_{*}, L_{*}\right)$ is stable. Furthermore, $\lambda(\tau)=0$ is always a root of characteristic equation (11). Thus the steady state $\left(K_{*}, L_{*}\right)$ is not asymptotically stable. 


\section{Application: Effect of Time Delay}

In this section, we study how the dynamics of the model (1) change when the time delay parameter varies.

Let's give the following numerical simulations:

Proposition 6 If $\alpha=0.5 ; \quad s=0.3 ; \quad A=1 ; \quad \delta=0.2 ; \quad \gamma=1 ; \quad$ and $g(K)=\frac{300 e^{K}}{1+e^{K}}$. Then system (1) have the following positive equilibrium

$$
E^{*}=(675 ; 300)
$$

Furthermore, the critical delay and the period of oscillations corresponding to (1) are $\tau_{0}=1.5861$ and $P_{0}=2 \pi$.

Proof. For

$$
\alpha=0.5 ; \quad s=0.3 ; \quad A=1 ; \quad \delta=0.2 \quad \gamma=1 \quad \text { and } \quad g(K)=\frac{300 e^{K}}{1+e^{K}},
$$

we have $f(K, L)=0.3 \sqrt{K L}$ and the system (1) becomes

$$
\left\{\begin{array}{l}
\frac{d K}{d t}=0.3 \sqrt{K L}-0.2 K \\
\frac{d L}{d t}=L\left[1-\frac{\left(1+e^{K}\right) L_{\tau}}{300 e^{K}}\right] .
\end{array}\right.
$$

System (19) has a unique positive equilibrium point $E^{*}=\left(K_{*} ; L_{*}\right)$ which satisfies the following conditions:

$$
L_{*}=\frac{4}{9} K_{*} \quad \text { and } \quad \frac{4}{9} K_{*}=\frac{300 e^{K}}{1+e^{K}} .
$$

Thus $E^{*}=(300 ; 675)$ (see Figure 1$)$.

The characteristic equation associated to system (19) is

$$
\lambda^{2}+0.1 \lambda+\lambda \exp (-\lambda \tau)+0.1 \exp (-\lambda \tau)=0
$$

which has a purely imaginary roots $\lambda_{0}= \pm 0.9987912 i$, for $\tau_{0}=\frac{1}{0.9987912} \cdot \frac{\pi}{2}=1.5719$.

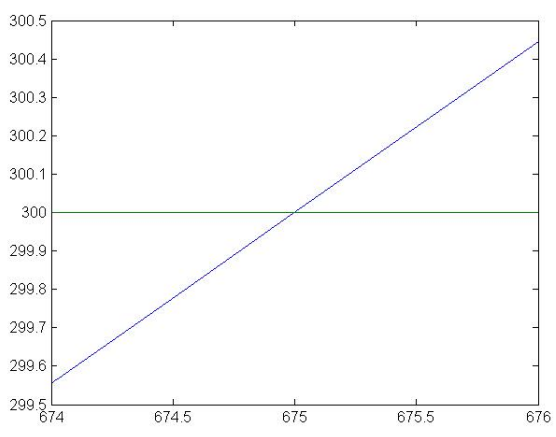

Figure 1. The graphs of functions $h_{1}(K):=\frac{4}{9} K$ and $h_{2}(K):=\frac{300 e^{K}}{1+e^{K}}$ on the interval $[674 ; 676]$.

\section{Conclusion}

In this paper, we propose a system of delay differential equations for modeling the interactions between the active population and the economic growth to explain economic growth and the unemployment phenomenon. When we take into account the time taken for the recruitment of the labor force, the cyclic behavior can appear for some specific values of parameters due to the Hopf bifurcation where the time delay parameter is the bifurcation parameter: for $\tau=1.4$, the time series of capital stock and employed workers are shown in Figure 2, and for $\tau=1.5719$, the Figure 3 describes periodic oscillations typical of real world economic variables. 

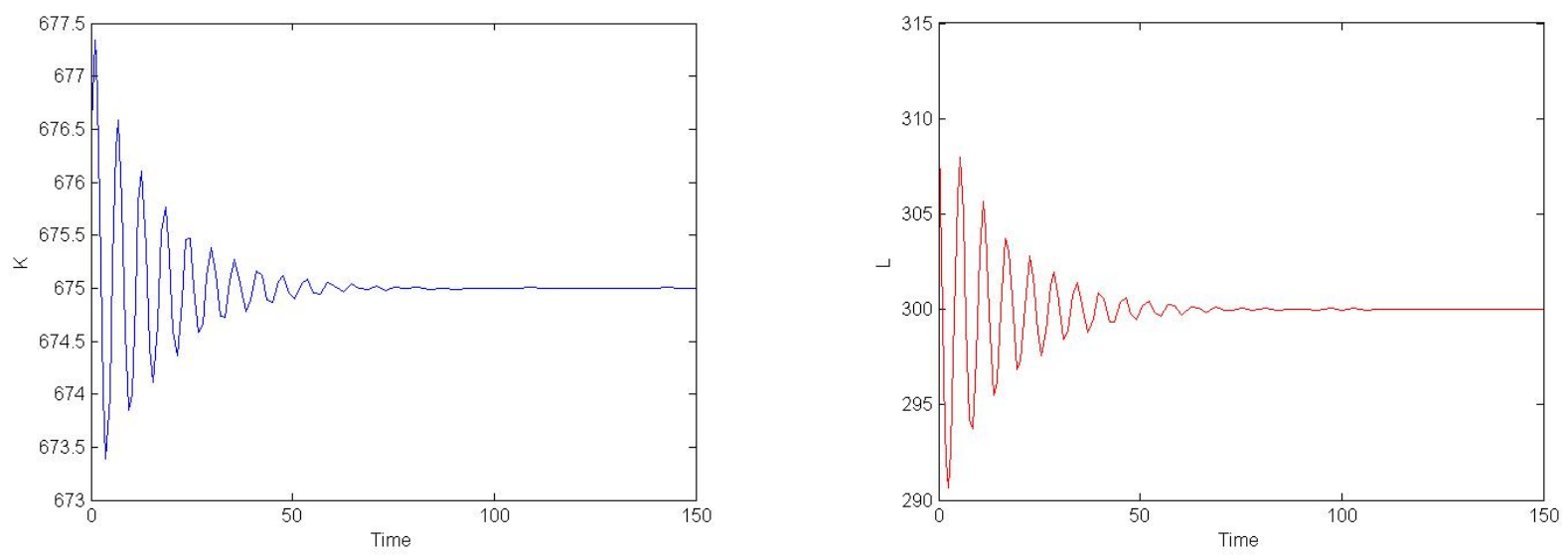

Figure 2. With the values given by Proposition 6, Solution of the model (1) is locally asymptotically stable for $\tau=1.4$. This solution has a period about $P_{0}=2 \pi$.
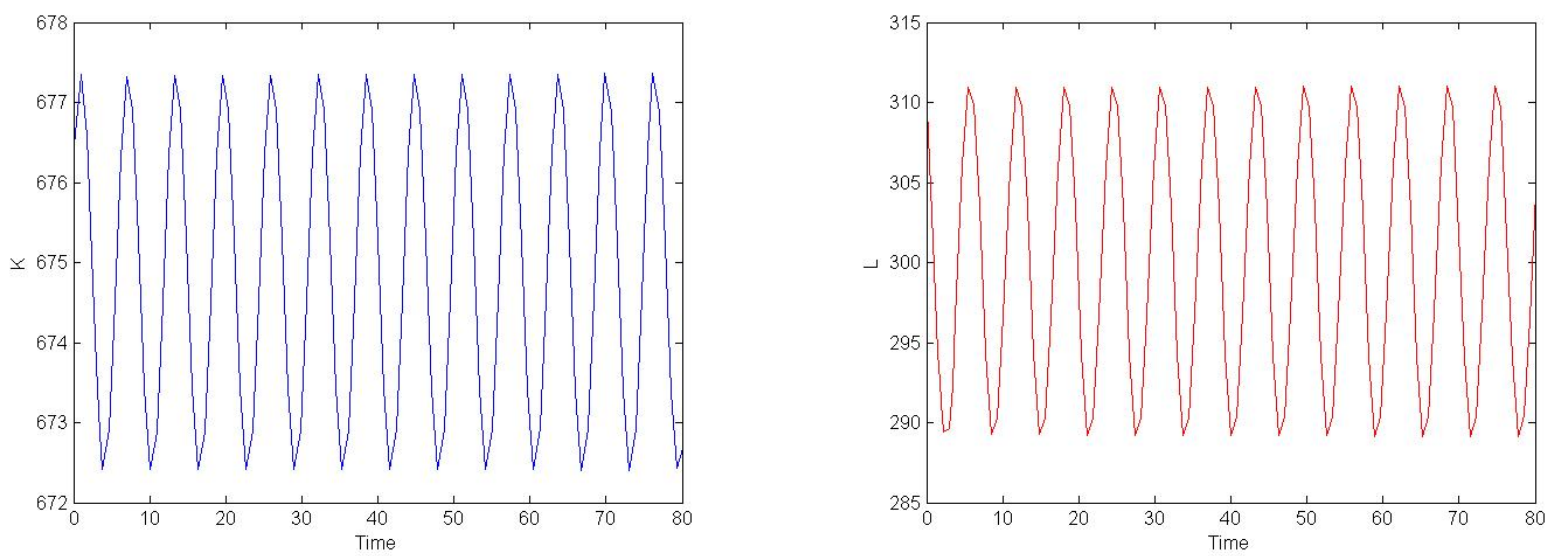

Figure 3. With the values given by Proposition 6, model (1) has a periodic solution for $\tau=1.5719$. This solution has a period about $P_{0}=2 \pi$.

\section{References}

1. R. Solow, "A contribution to the theory of economic growth," The Quarterly Journal of Economics, vol. 70, no. 1 , pp. 65-94, 1956.

2. S. Hallegatte, M. Ghil, P. Dumas, and J.-C. Hourcade, "Business cycles, bifurcations and chaos in a neoclassical model with investment dynamics," Journal of Economic Behavior and Organization, vol. 67, no. 1, pp. 57-77, 2008.

3. D. Cai, "An economic growth model with endogenous carrying capacity and demographic transition," Mathematical and Computer Modelling, vol. 55, no. 1, pp. 432-441, 2012.

4. C. Cobb and P. Douglas, "A theory of production," The American Economic Review, vol. 18, no. 1, pp. 139-165, 1928.

5. R. Harrod, "An essay in dynamic theory," The Economic Journal, vol. 49, no. 193, pp. 14-33, 1939.

6. E. D. Domar, "Expansion and employment," The American Economic Review, vol. 37, no. 1, pp. 34-55, 1947.

7. D. Cai, "Multiple equilibria and bifurcations in an economic growth model with endogenous carrying capacity," International Journal of Bifurcation and Chaos, vol. 20, no. 11, pp. 3461-3472, 2010.

8. L. Guerrini and M. Sodini, "Nonlinear dynamics in the solow model with bounded population growth and time-to-build technology," Abstract and Applied Analysis, vol. 2013, no. Article ID 836537, p. 6 pages, 2013.

9. M. Kalecki, "A macrodynamic theory of business cycles," Econometrica, vol. 3, no. 3, pp. 327-344, 1935. 
10. L. Guerrini, "Analysis of an economic growth model with variable carrying capacity," International Journal of Mathematical Analysis, vol. 7, no. 26, pp. 1263-1270, 2013.

11. J. Dieudonne, "Foundations of modern analysis," 1960.

12. J. K. Hale, "Introduction to functional differential equations," 1993. 